\title{
Energy Expenditure of Common Sedentary Activities in Youth
}

\author{
Melissa Lau, Li Wang, Sari Acra, Maciej S. Buchowski
}

\begin{abstract}
Background: Standardized measures of energy expenditure (EE) for sedentary activities in youth are needed. The goal was to determine EE of common contemporary and computer-related sedentary activities in youth. Methods: We measured EE for sedentary tasks in 10 - to 17 -year-old youths $(n=24)$ during $\sim 24$ hours in a whole-room indirect calorimeter. Directly monitored tasks were performed for $\sim 10$-min. EE was calculated from oxygen consumed and carbon dioxide produced, converted to metabolic equivalents (MET) by normalization to an individual's measured resting EE, and compared with the Compendium of Energy Expenditures for Youth. Results: Compared with the youth compendium, measured METs were lower for internet surfing (1.3), computer keyboard typing (1.3), and sorting beads/crafts (1.5) (all $P<.002$ ), and similar for handwriting (1.4), playing cards (1.6), video-gaming (1.6), and telephoning (1.5). Conclusions: Current youth compendium MET estimates should be used with caution when predicting EE of common contemporary and computer-related sedentary activities in youth.
\end{abstract}

Keywords: sedentary behavior, physical activity, accelerometry, measurement

Physical inactivity has been implicated as a major contributor to the obesity epidemic worldwide. In the US, $18 \%$ of children age 6 to 11 years and $21 \%$ of adolescents age 12 to 19 years were obese in 2012. ${ }^{2}$ US national guidelines currently recommend at least 60 minutes of moderate-to-vigorous aerobic activity daily, with a minimum 3 days/week dedicated to vigorous-intensity activities, defined as 7 to 8 of 10 in subjective intensity scales. ${ }^{6}$ Thus, proper characterization of physical activity (PA) patterns and energy expenditure (EE) in y outh is crucial for comparing individual health-related behaviors to public health recommendations.

In epidemiological and clinical studies, EE is commonly a ssessed using self-reported activities and corresponding metabolic equivalents of tasks (METs), defined as the ratio of the metabolic rate for a particular activity normalized to an individual's resting energy expenditure (REE). ${ }^{1}$ MET values are considered an accurate means of assessing PA-related EE (PAEE) and assigning intensity categories to sedentary and active behaviors. Compendia of MET values were developed for adults ${ }^{1}$ and, more recently, for youth. ${ }^{7}$ Compendia report a single MET value for each activity, averaged for age, sex, gender, and other characteristics. The Compendium of Energy Expenditures for Youth (youth compendium) is considered a work in progress, as approximately $65 \%$ of the activities have MET estimates based on the adult compendium with remaining values based on limited empirical data. ${ }^{4}$ The reliability of such estimates must be verified in various populations and must consider physiological factors unique to youth (eg, puberty or body composition) that alter REE and PAEE. In addition, MET values for some sedentary activities routine for youths, such as internet searching or typing, have not been reported.

Lau is with the Albert Einstein School of Medicine. Wang is with the Dept of Biostatistics, Vanderbilt University Medical Center. Acra is with the Dept of Pediatrics, Vanderbilt University Medical Center. Buchowski (maciej. buchowski@vanderbilt.edu) is with the Energy Balance Laboratory, Division of Gastroenterology, Hepatology, and Nutrition, Dept of Medicine, Vanderbilt University.
Thus, our objective was to measure oxygen $\left(\mathrm{O}_{2}\right)$ uptake, estimate EE costs, and generate MET estimates for some common sedentary activities in youth using whole-room indirect calorimetry. We compared these empirical results to similar activities reported in the youth compendium.

\section{Methods}

\section{Participants}

Healthy youth ( $\mathrm{n}=24,12$ females, age 10 to 17 years) were recruited to a bigger study evaluating the accuracy of accelerometry in predicting PAEE in free-living youth across a range of body mass indices (BMI, kg/m²) in an age-, sex-, and ethnicity-stratified group of children in Nashville, Tennessee. Recruitment strategies included flyers, emails, and personal contacts. Inclusion criteria included being healthy as assessed by a pediatrician, nonsmoking, not having limitations on exercise tolerance or chronic pulmonary conditions (eg, asthma), and not taking prescription medications interfering with EE. Before the study, participating youths and their parents or guardians signed an informed consent or assent approved by the Vanderbilt University Institutional Review Board. Participants were encouraged to maintain normal daily activities but avoid unusual or strenuous activities the day before the study in the Clinical Research Center.

\section{Study Design and Protocol}

Study participants spent $\sim 24$ hours in a fast response, whole-room indirect calorimeter (volume: $19 \mathrm{~m}^{3}$ ) that assures high-precision EE measurements in a controlled environment under seminaturalistic conditions (eg, not wearing a breathing mask). A detailed description and the accuracy of our room calorimeter for measuring EE were reported previously. ${ }^{8}$ The room is airtight with windows and equipped with toilet, sink, desk and chair, telephone, multimedia, and exercise equipment. Investigators communicated with participants through intercom. During the stay, participants followed a structured protocol involving self-paced, 10-minute ambulatory 
and sedentary tasks throughout morning and afternoon sessions, and has been described previously. ${ }^{8}$ Sedentary tasks were handwriting, playing cards, video-gaming, sorting beads/crafts, talking on the phone, typing, and internet surfing (see Table 2). Each task was followed by a 10-minute rest, allowing EE to return to steady state before starting the next activity. A trained research assistant observed the session through the room calorimeter window, marking the start and end of each task using a computerized system timesynchronized with the room calorimeter. Meals (breakfast, lunch, dinner, afternoon and evening snacks) were given at set times and contained macro- and micronutrient content individualized for body weight and activity level. Participants were instructed to go to bed at 10:00 PM and wake up at 6:00 AM to measure REE.

\section{Measurements}

Anthropometrics. Stature (height) was measured $( \pm 0.5 \mathrm{~cm})$ using a calibrated wall-mounted stadiometer (Perspective Enterprises, Portage, MI) and body weight $( \pm 0.1 \mathrm{~kg})$ using a calibrated scale (Detecto-Medic, Detecto Scales, Inc, Northbrook, IL) with participants wearing light clothing and no shoes. Body mass index (BMI, $\mathrm{kg} / \mathrm{m}^{2}$ ) and BMI percentiles were calculated from height, weight, age, and gender using Centers for Disease Control (CDC) growth charts. Total fat and fat-free mass $(\mathrm{kg})$ were measured using dual energy x-ray absorptiometry (DXA), (GE Medical Systems, Madison WI, enCORE 2007 software v.11.40.004).

Energy Expenditure (EE, kcal/min). Total EE was calculated minute-by-minute from measured rates of $\mathrm{O}_{2}$ consumption and carbon dioxide $\left(\mathrm{CO}_{2}\right)$ production using Weir's equation. ${ }^{9}$

Resting EE (REE, kcal/min). REE was calculated as the average EE measured during a 30-min period while the participant laid quietly in bed the morning following an overnight sleep and 10-hour fast, as described previously. ${ }^{8}$

EE of Sedentary Tasks (PAEE, kcal/min). PAEE for each task was calculated as an average EE during the time ( 10-min) the activity was performed.

MET Values for Sedentary Tasks. MET values for each task were calculated as the ratio of measured PAEE and measured REE for each participant.

\section{Statistical Analysis}

Descriptive characteristics are presented as the median with interquartile range (IQR) for continuous variables and as frequencies and percentages for categorical variables. Average measured METs for each activity was compared with similar intensity activities from both compendia using one-sample $t$ test. Differences in personal characteristics and MET values between genders were analyzed using Wilcoxon test. All tests were two-tailed with 5\% significance level. Analyses were performed using R statistical software (version 3.1.2, Vienna, Austria).

\section{Results}

\section{Personal Characteristics}

There were no significant differences between genders; thus, combined data are presented (Table 1). According to BMI categorization, ${ }^{2}$ approximately half the participants were overweight or obese, and half had normal weight with median BMI of $25.5 \mathrm{~kg} / \mathrm{m}^{2}$ (IQR
Table 1 Demographic and Anthropometric Characteristics of Study Participants $\left(n=24,12\right.$ Females $\left.^{a}\right)$

\begin{tabular}{lc}
\hline Variable & IQR $^{\mathbf{b}}$ \\
\hline Age (years) & $13(13-16)$ \\
Weight $(\mathrm{kg})$ & $69(59-79)$ \\
Height $(\mathrm{cm})$ & $162(156-170)$ \\
Body mass index $\left(\mathrm{BMI}, \mathrm{kg} / \mathrm{m}^{2}\right)$ & $25.5(23.8-31.1)$ \\
BMI percentile & $95(87.3-98.0)$ \\
Fat free mass $(\mathrm{kg})$ & $44.5(38.0-51.5)$ \\
Body fat $(\%)$ & $36.0(33.1-40.2)$ \\
\hline
\end{tabular}

a Ethnicity: 11 black, 1 Hispanic, 12 white.

${ }^{\mathrm{b}}$ Values are median (lower-upper) interquartile ranges (IQR).

${ }^{\mathrm{c}}$ Calculated using Centers for Disease Control (CDC) growth charts.

23.8-31.1) and BMI percentile of 95 (IQR 87.3-98.0). Fat-free mass was slightly higher in males than females $(P=.24)$.

\section{REE and PAEE (METs)}

Oxygen uptake, REE and PAEE are in Table 2. The average oxygen uptake ranged from $3.7 \pm 0.7 \mathrm{~mL} / \mathrm{kg} / \mathrm{min}$ at rest to $7.26 \pm 2.88 \mathrm{~mL} /$ $\mathrm{kg} / \mathrm{min}$ for video-gaming; EE ranged from $1.30 \pm 0.23 \mathrm{kcal} / \mathrm{min}$ at rest to $2.14 \pm 0.51 \mathrm{kcal} / \mathrm{min}$ for video-gaming. MET values ranged from $1.32 \pm 0.14$ for typing to $1.62 \pm 0.25$ for video-gaming. The average MET values were $1.43 \pm 0.14$ and $1.42 \pm 0.11$ for all combined and computer-related sedentary tasks, respectively.

\section{Comparison of Measured METs With the Youth Compendium}

There were significant differences between our measured MET values for sorting beads (1.46), video-gaming (1.6), typing (1.32) and internet surfing (1.33) compared with the corresponding youth compendium values $(1.6,1.5,1.8$ and 1.8 METs respectively, all $P<.002)$. Differences between measured and youth compendium MET values for handwriting, playing cards, and talking on the phone were not significant (all $P>.05$ ). Of note, reference values for typing, internet surfing and talking on the phone were derived from adult compendium values. ${ }^{7}$

There were differences in MET values between males and females for video-gaming $(1.7 \pm 0.2$ and $1.5 \pm 0.3$ respectively, $P$ $=.039)$ and sorting beads $(1.5 \pm 0.2$ and $1.4 \pm 0.2$ respectively, $P$ $=.021)$ but not handwriting, playing cards, talking on the phone, typing, or internet surfing (all $P>.05$ ).

\section{Discussion}

In this study, we determined PAEE of common sedentary activities in 10- to 17-year-old youth with a broad range of BMI percentiles using the reference standard of indirect room calorimetry. To adequately compare these EE with other studies and with the reference youth compendium, we calculated MET values as a ratio of measured EE for each task to REE.

Our results are consistent with other studies, which reported similar MET ranges (1.2 to 1.6) for activities equivalent to handwriting/homework reading, playing board games, crafting, and seated video-gaming in youth. ${ }^{3,4}$ 


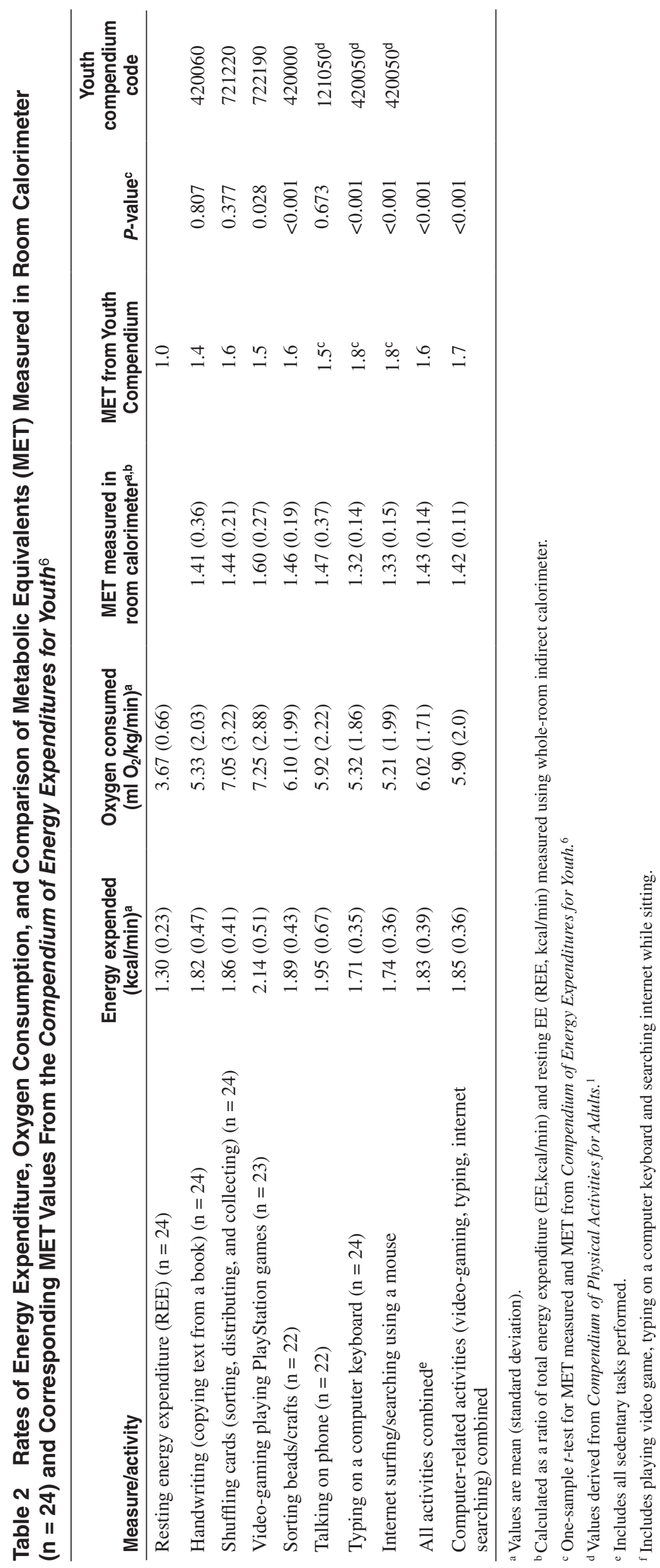


Our results show that youth compendium MET estimates for sedentary activities derived from adult values (typing and internet surfing) overestimate true EE in youth; these findings are consistent with results from other studies. ${ }^{3,5}$ The discrepancy might be caused in part by well-known differences in energy cost of PA between youth and adults, as well as relatively higher REE in youth. ${ }^{5}$ In our study, the average REE was $3.67 \mathrm{~mL} / \mathrm{kg} / \mathrm{min}$, which is higher than $3.50 \mathrm{ml} / \mathrm{kg} / \mathrm{min}$ used in both youth and adult compendia. It has been suggested the youth compendium be adjusted for higher REE in children. ${ }^{5}$

We propose that our study's MET values for typing (1.32), internet surfing (1.33), and combined computer activities (1.42) should be considered when updating the youth compendium.

Our study is strengthened by the use of indirect room calorimetry, allowing for accurate $\mathrm{O}_{2}$ consumption and $\mathrm{CO}_{2}$ production measurements in a seminaturalistic environment (ie, without facemask) that would otherwise impact movement during the activity tasks. Our MET calculations relied on REE measured in the room calorimeter instead of the reference value of $3.50 \mathrm{ml} / \mathrm{kg} / \mathrm{min}$. We also included study subjects across a wide range of BMIs, age, and ethnicities.

Study limitations include a small sample size $(n=24)$, limiting generalizability to other populations, especially younger children, and limiting the power to assess differences between genders. Despite this small sample size, our investigation into youth populations is valuable, as the adult and youth compendia had not derived a majority of MET estimates from youth.

While the clinical and epidemiological implications of our findings remain unknown, we encourage further studies to estimate PAEE for specific sedentary behaviors, which may be of particular importance given the increasing popularity of electronic devices that promote sedentary lifestyles.

We conclude that current youth compendium MET estimates, especially those derived from the adult compendium, should be used with caution when predicting EE of common contemporary and computer-related sedentary activities in youth

\section{Acknowledgments}

The authors thank Marissa Avolio, Stephan Daphnis, Cindy Dorminy, Kristen Jensever, Natalie Meade, and Elizabeth Provenzano for their contributions to data acquisition. This work was supported by grants RO1-HL082988, UL1-RR024975, DK20593, and T35-DK007383 from the National Institutes of Health.

\section{References}

1. Ainsworth BE, Haskell WL, Herrmann SD, et al. 2011 Compendium of Physical Activities: a second update of codes and MET values. Med Sci Sports Exerc. 2011;43:1575-1581 doi:10.1249/ MSS.0b013e31821ece12. PubMed

2. Centers for Disease Control and Prevention. The CDC Guide to Strategies to Increase Physical Activity in the Community. 2015. cdc.gov/ obesity/downloads/PA_2011_WEB.pdf.

3. Harrell JS, McMurray RG, Baggett CD, et al. Energy cost of physical activities in children and adolescents. Med Sci Sports Exerc. 2005;37:329-336. PubMed doi:10.1249/01. MSS.0000153115.33762.3F

4. Lyden K, Keadle SK, Staydenmayer J, et al. Energy cost of common activities in children and adolescents. J Phys Act Health. 2013;10:6269. PubMed

5. McMurray RG, Butte NF, Crouter SE, et al. Exploring metrics to express energy expenditure of physical activity in youth. PLoS One. 2015;10(6):e0130869 doi:10.1371/journal.pone.0130869. PubMed

6. Ridley K, Ainsworth BE, Olds TS. Development of a compendium of energy expenditures for youth. Int J Behav Nutr Phys Act. 2008;5:45. PubMed doi:10.1186/1479-5868-5-45

7. Sun M, Reed GW, Hill JO. Modification of a whole room indirect calorimeter for measurement of rapid changes in energy expenditure. J Appl Physiol. 1994;76:2686-2691. PubMed

8. Warolin J, Carrico AR, Whitaker LE, et al. Effect of BMI on prediction of accelerometry-based energy expenditure in youth. Med Sci Sports Exerc. 2012;44:2428-2435. PubMed doi:10.1249/ MSS.0b013e318267b8f1

9. Weir J. New methods for calculating metabolic rate with special reference to protein metabolism. J Physiol. 1949;109:1-9. PubMed doi:10.1113/jphysiol.1949.sp004363 